\title{
Thickness of Bar 1-Visibility Graphs
}

\author{
Stefan Felsner and Mareike Massow \\ Technische Universität Berlin, Fachbereich Mathematik \\ Straße des 17. Juni 136, 10623 Berlin, Germany \\ \{felsner, massow\}@math.tu-berlin.de
}

\begin{abstract}
Bar $k$-visibility graphs are graphs admitting a representation in which the vertices correspond to horizontal line segments, called bars, and the edges correspond to vertical lines of sight which can traverse up to $k$ bars. These graphs were introduced by Dean et al. 3] who conjectured that bar 1-visibility graphs have thickness at most 2 . We construct a bar 1-visibility graph having thickness 3, disproving their conjecture. For a special case of bar 1-visibility graphs we present an algorithm partitioning the edges into two plane graphs, showing that for this class the thickness is indeed bounded by 2 .
\end{abstract}

\section{Introduction}

Visibility is a major topic in discrete geometry where a wide range of classes of visibility graphs has been studied, see e.g. [1], [2], 5], [9], [10]. Among the best studied variants are the traditional bar visibility graphs, they admit a complete characterization which has been obtained independently by Wismath [14 and Tamassia and Tollis [13. On the previous Graph Drawing Symposium, Dean, Evans, Gethner, Laison, Safari and Trotter [3, 4, introduced the class of bar $k$ visibility graphs $(\mathrm{B} k \mathrm{Vs})$ which 'interpolates' between two classes of graphs with a representation by a family of intervals, namely between bar visibility graphs and interval graphs. Dean et al. are mainly interested in measurements of closeness to planarity of bar $k$-visibility graphs. They prove a bound of 4 for the thickness of bar 1-visibility graphs and conjecture that they actually have thickness at most 2. In Sect. 2 we disprove this conjecture by showing that there are bar 1-visibility graphs with thickness 3. In Sect. 3 we attack the problem from the other side. We consider bars which extend from the $y$-axis to the right, such bars are called semi bars. The class of semi bar 1-visibility graphs (SB1Vs) is shown to have thickness 2 , the proof is based on an algorithm that partitions the edges of a given SB1V into two planar graphs. In the remainder of this introduction we make the terminology more precise.

\section{$1.1 \quad$ Thickness}

Thickness is a parameter that measures how far a graph is from being planar: The (graph-theoretic) thickness of a graph $G$, denoted by $\theta(G)$, is defined as the minimum number of planar subgraphs whose union is $G$. 
Determining the thickness of a graph is NP-hard (see [11]). Exact values are only known for very few classes of graphs. For a survey on theoretical and practical aspects see 12 .

Note that in the definition of thickness, the planar embeddings of the subgraphs do not have to coincide. The geometric thickness of $G$ asks for the minimum number of subgraphs/colors in the following setting: Choose a straightline embedding of $G$ and a coloring of the edges such that crossing edges have different colors, the edges of each color then form a plane graph. Geometric thickness was introduced by Dillencourt, Eppstein and Hirschberg in [6]. In [7], Eppstein showed that graph-theoretic thickness and geometric thickness are not even asymptotically equivalent.

\subsection{Bar $k$-Visibility Graphs}

Let a collection of pairwise disjoint horizontal line segments (called bars) in the Euclidean plane be given. Construct a graph based on these bars as follows: Take a set of vertices representing the bars. Two vertices are joint by an edge iff there is a line of sight between the two corresponding bars (we then say that the bars see each other). A line of sight is a vertical line segment connecting two bars and intersecting at most $k$ other bars. A graph is a bar $k$-visibility graph $(\mathrm{B} k \mathrm{~V})$ if it admits such a bar representation.

We call the lines of sight that don't intersect any bar direct, all others are indirect lines of sight; we also use these adjectives for the corresponding edges.
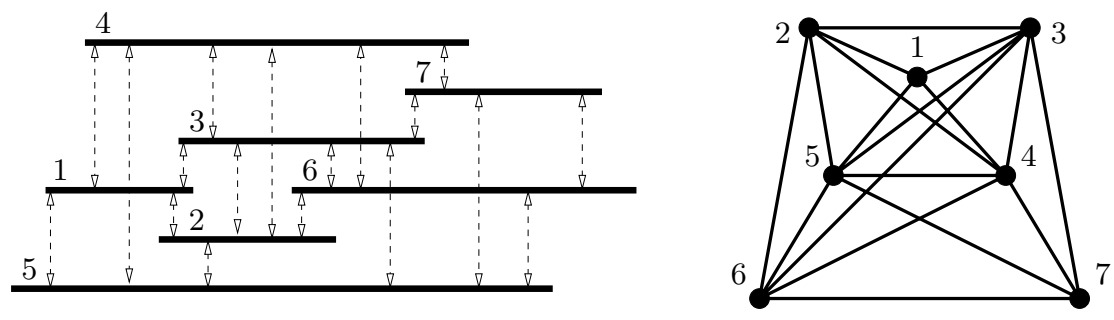

Fig. 1. Example of a bar 1-visibility graph

Note that bar visibility graphs can be regarded as bar 0-visibility graphs, and interval graphs as bar $\infty$-visibility graphs. Given a bar representation, we can consider the induced $\mathrm{B} k \mathrm{~V}$ for any $k$. In the following, we will - unless otherwise mentioned - only deal with the case $k=1$. Figure 1 shows an example of a B1V where lines of sight are indicated by dashed lines.

Throughout this paper, we assume that all bars are located at different heights. This can easily be obtained by slightly altering the $y$-coordinates of some bars. We also assume all endpoints of bars to have pairwise different $x$ coordinates by slightly permutating the $x$-coordinates of the endpoints in a given bar representation. (This might result in additional edges, but since we consider problems that only get harder when the number of edges increases, our results 
extend to general $\mathrm{B} k \mathrm{Vs}$.) Note that with this assumption, lines of sight can be thougth of as pillars of positive width.

A semi bar $k$-visibility graph $(\mathrm{SB} k \mathrm{~V})$ is a bar $k$-visibility graph admitting a representation in which all left endpoints of the bars are at $x=0$.

Note that for $k=0$, these graphs have been investigated in [2] where they are identified as the graphs of representation index $1+1 / 2$.

For the class of $\mathrm{SB} k \mathrm{Vs}$ the assumption that all endpoints of bars have different $x$-coordinates only refers to the right endpoints. For convenience, we rotate semi bar representations of $\mathrm{SB} k \mathrm{Vs}$ counterclockwise as shown in Fig. 2, in the following we will always think of semi bar representations in this way.
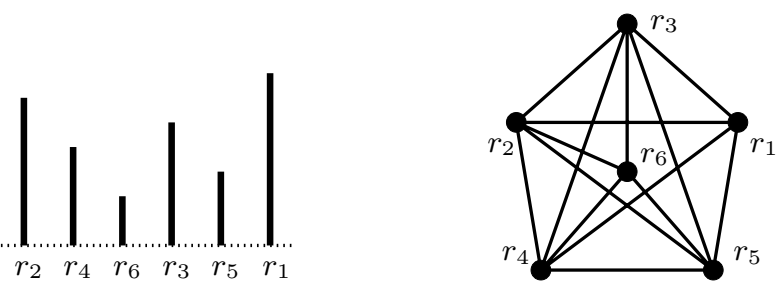

Fig. 2. Example of a semi bar 1-visibility graph

Label the bars of a semi bar representation $r_{1}, r_{2}, \ldots, r_{n}$ by decreasing $y$ coordinate of the upper endpoint, i.e., by decreasing height. Reading these labels from left to right we obtain a permutation of $[n]$ which completely determines the graph. The SB1V from the figure is encoded by the permutation $(2,4,6,3,5,1)$.

\section{A Bar 1-Visibility Graph with Thickness 3}

In [3, Dean et al. used the Four Color Theorem to show that the thickness of $\mathrm{B} 1 \mathrm{Vs}$ is bounded by 4 . They conjectured that the correct bound is 2 . In this section we construct a B1V with thickness 3 . We will often talk about a 2coloring of a graph $G=(V, E)$, meaning a 2-coloring of the edges such that each color class is the edge set of a planar graph on $V$. Given a 2-coloring (with blue and red) we define $G_{\text {blue }}$ and $G_{\text {red }}$ as the graphs on $V$ with all blue and all red edges, respectively.

Here is a brief outline of the construction: First we analyze a quite simple type of graph which has thickness 2 but with the property that every 2-coloring has uniform substructures, so called lampions. Assuming that the original graph is large enough we can assume arbitrarily large lampions. In a second step we introduce a series of slight perturbations into the original graph. It is shown that most of these perturbations have to be incorporated into lampions and the number of perturbations in one lampion is proportional to its size. However a lampion can only absorb a constant number of the perturbations. This yields a contradiction to the assumption that a 2-coloring exists. 
We start with an Autobahn where we have heaped up the median strip: Consider the bar representation of the graph $A_{n}$ shown in Fig. 3. This graph has four outer vertices $A, B, C, D$ and a set $V_{\text {inner }}$ of $n$ inner vertices.
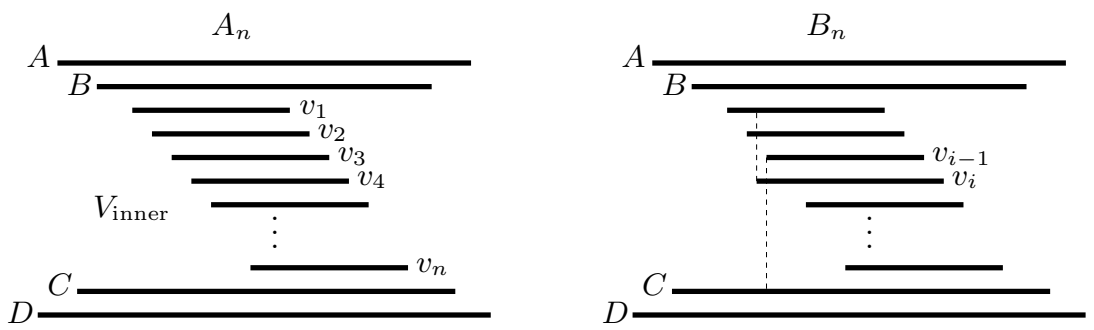

Fig. 3. The Autobahn-graph $A_{n}$ and its modified counterpart $B_{n}$

Since $A_{n}$ contains a $K_{4, n}$ as subgraph we know that $A_{n}$ is non-planar (assuming $n \geq 3$ ), hence, $\theta\left(A_{n}\right) \geq 2$. To show that $\theta\left(A_{n}\right)=2$ we let $G_{\text {blue }}$ consist of all direct edges and $G_{\text {red }}$ consist of all indirect edges. Figure 4 shows the partition.
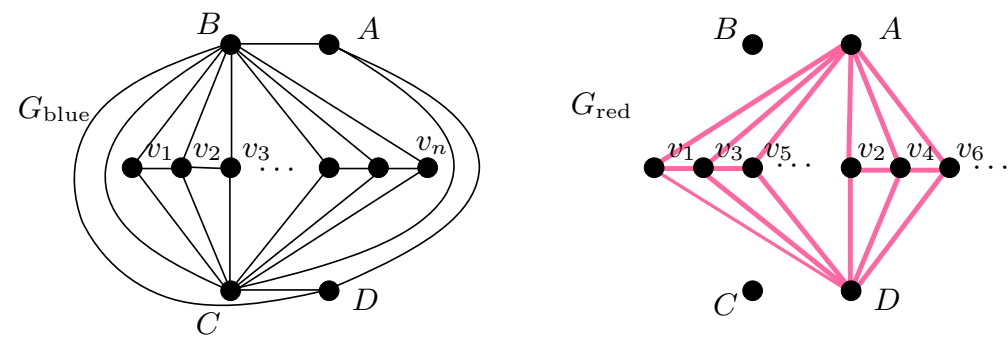

Fig. 4. A partition of $A_{n}$ into two planar graphs

Let $V_{\text {inner }}=\left\{v_{1}, v_{2}, \ldots, v_{n}\right\}$, such that the indices represent the order of the right endpoints of the bars from left to right. The inner neighbors of $v_{i}$ are $v_{i-2}, v_{i-1}, v_{i+1}$ and $v_{i+2}$. The graph $G\left[V_{\text {inner }}\right]$ induced by the inner vertices is maximal outerplanar with an interior zig-zag.

A lampion in a 2-coloring of $A_{n}$ consists of a set $W=\left\{v_{i}, v_{i+1}, \ldots v_{j}\right\}$ of consecutive inner vertices and a partition $\left\{S_{1}, S_{2}\right\},\left\{S_{3}, S_{4}\right\}$ of the four outer vertices such that $G_{\text {blue }}$ consists of all zig-zag edges of $G[W]$ and all edges connecting vertices from $W$ with $S_{1}$ and $S_{2}$, while $G_{\text {red }}$ consist of the two outer

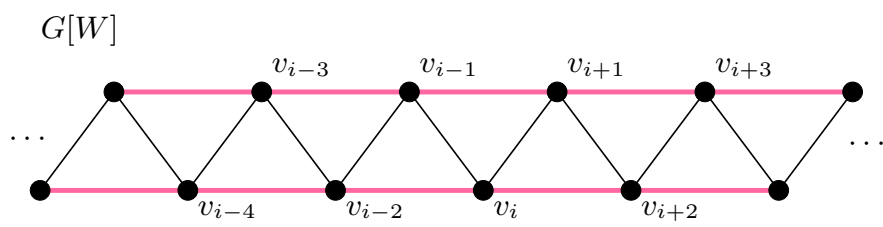

Fig. 5. A lampion coloring of $G[W]$ 
paths of $G[W]$ and all edges connecting vertices from $W$ with $S_{3}$ and $S_{4}$ (of course exchanging red and blue again yields a lampion). The set $W$ is the core of the lampion. Thus, Fig. 4 shows a lampion with core $V_{\text {inner }}$ together with the additional edges between the four outer vertices.

Lemma 1. For every $k \in \mathbb{N}$ there is an $n \in \mathbb{I N}$ such that in every 2-coloring of $A_{n}$ there is a $W \subset V_{\text {inner }}$ with $|W| \geq k$ such that $W$ is the core of a lampion.

Proof. Each inner vertex has four outer neighbors. Let's call an edge connecting an inner and an outer vertex a transversal edge. Consider the blue transversal edges; at each vertex there can be $0,1,2,3$ or 4 of them. But $G_{\text {blue }}$ is planar and therefore does not contain a $K_{3,3}$. Thus, at most two inner vertices can have the same three outer neighbors in $G_{\text {blue }}$. There are $\left(\begin{array}{l}4 \\ 3\end{array}\right)=4$ different triples of outer neighbors in $G_{\text {blue }}$, so there can be at most eight inner vertices with more than two outer neighbors in $G_{\text {blue }}$. We might find another eight in $G_{\text {red }}$. These irregular vertices break the sequence $v_{1}, v_{2}, v_{3}, \ldots, v_{n}$ of inner vertices of $A_{n}$ into at most 17 pieces. By pigeon-holing, there must be at least one piece with size $n^{\prime} \geq(n-16) / 17$ such that in the induced 2-coloring of $A_{n^{\prime}}$ all inner vertices are incident to exactly two blue and two red transversal edges.

Considering only the blue transversal edges of $A_{n^{\prime}}$, the resulting subgraph $G_{\text {blue }}^{\prime}$ is a subgraph of a blown-up $K_{4}$ as illustrated in Fig. 6. This subgraph is not arbitrary but has the property that every inner vertex has exactly two incident edges.

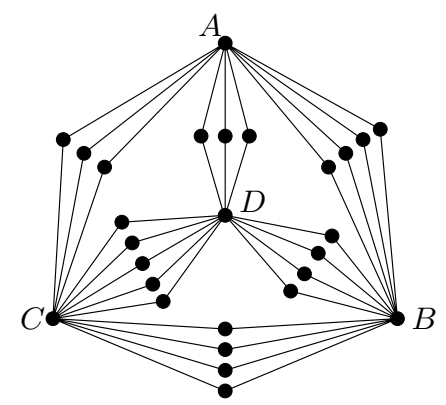

Fig. 6. Blown-up $K_{4}$

Now it remains to planarly embed the inner edges of $A_{n^{\prime}}$, i.e. those of $G\left[V_{\text {inner }}^{\prime}\right]$. To our disposition we have the $\leq 4$ 'large faces' of the blown-up $K_{4}$, which makes eight faces in total for the two planar graphs. In each of these faces we can embed at most three inner edges. There are other cases with fewer large faces which have in turn more inner vertices at the boundary. In all cases it is impossible to embed more than 12 edges between inner vertices with different outer neighbors in $G_{\text {blue }}^{\prime}$, the red subgraph may contain another 12 irregular edges. These at most 24 irregularities break the sequence of inner vertices of $A_{n^{\prime}}$ into at most 25 pieces, we remain with a 2 -coloring of $A_{n^{\prime \prime}}$ with $n^{\prime \prime} \geq\left(n^{\prime}-24\right) / 25$ such that all inner vertices are incident to the same two outer vertices in $G_{\text {blue }}^{\prime}$ and to the other two outer vertices in $G_{\text {red }}^{\prime}$. 
We now have $K_{2, n^{\prime \prime}}$ as a subgraph in both $G_{\text {blue }}^{\prime \prime}$ and in $G_{\text {red }}^{\prime \prime}$. The good thing about this is that $K_{2, n^{\prime \prime}}$ has an (essentially) unique planar embedding. Consequences for the inner edges of $A_{n^{\prime}}$ are exploited in the following facts.

Fact 1. Every inner vertex of $A_{n^{\prime \prime}}$ has at most two incident inner edges of each color.

It follows that the 2-coloring of $A_{n^{\prime \prime}}$ induces a 2-coloring of $G\left[V_{\text {inner }}^{\prime \prime}\right]$ such that each color consists of a set of paths and cycles.

Fact 2. The set of blue inner edges of $A_{n^{\prime \prime}}$ contains at most one cycle. The same holds for the red inner edges. If there is a monochromatic cycle, then it is a spanning cycle of $V_{\text {inner }}^{\prime \prime}$.

There is not much freedom for a 2-coloring of the inner edges of $A_{n^{\prime \prime}}$ with these properties: We almost have a lampion coloring on $G\left[V_{\text {inner }}^{\prime \prime}\right]$. The exception is that there can be a single Z-structure (see Fig. 7) in one color.

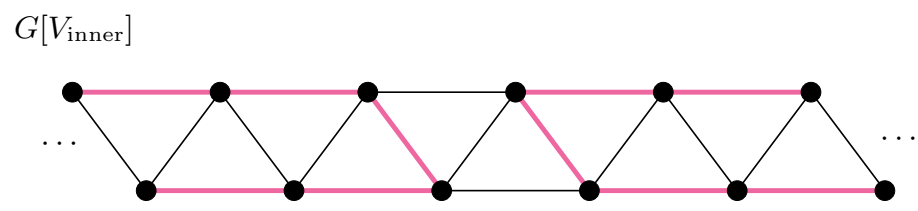

Fig. 7. One Z-structure and no monochromatic cycle force all other edge-colors

Removing the Z-structure leaves two consecutive pieces of the sequence of inner vertices. These pieces of $V_{\text {inner }}^{\prime \prime}$ have a lampion coloring. The size of the larger piece can be estimated as $n^{\prime \prime \prime} \geq\left(n^{\prime \prime}-4\right) / 2$. This proves the lemma.

Well-prepared we can now look at the variant $B_{n}$ of $A_{n}$ in which we have slightly perturbed some of the inner bars (see Fig. 3 ). To get $B_{n}$, we have elongated every (say) tenth inner bar by pulling its left endpoint to the left, such that it is further left than the left endpoint of the bar directly above. With this modification we introduce an additional edge between the elongated bar $v_{i}$ and $v_{i-3}$, but in turn we lose the edge between the bar $v_{i-1}$ and the lowest outer bar $D$. Let's call the vertices corresponding to the elongated bars modified vertices.

Theorem 1. The graph $B_{n}$ is a bar 1-visibility graph with thickness 3 , for $n$ large enough.

Proof. We will show that $B_{n}$ has no 2-coloring. It follows that its thickness is at least 3 , and since we can easily use a 2-coloring of $A_{n}$ and embed the independent additional edges in a third graph, $B_{3}$ has thickness exactly 3 .

Assume that $B_{n}$ admits a 2-coloring. We first show that any 2-coloring of $B_{n}$ would have to be very much alike a 2-coloring of $A_{n}$.

The vertices corresponding to a bar directly above a modified one - let's call them reduced - have only three outer neighbors. To avoid a $K_{3,3}$ in one color there can be at most 16 inner vertices incident to more than two transversal edges. In particular, most reduced vertices have to divide their three incident 
transversal edges into two of one color and one of the other. As in the proof of the lemma we consider a continuous piece in the sequence of inner vertices such that all inner vertices have at most two transversal edges of each color. The graph induced by the largest of these pieces and the outer vertices is $B_{n^{\prime}}$.

The blue subgraph $G_{\text {blue }}^{\prime}$ of $B_{n^{\prime}}$ is a subgraph of a blown-up $K_{4}$ where at least $9 / 10$ of the inner vertices have degree 2 and the remaining reduced vertices have degree 1 . As in the proof of the lemma it can be argued that there is only a constant number $c$ of edges in $G_{\text {blue }}^{\prime}$ which join two inner vertices such that there are at least three different outer neighbours of these two vertices, i.e., which join two inner vertices which not belonging to the same blown-up edge of $K_{4}$. The constant can be bounded as $c \leq 24$. The red graph may contribute another set of $c$ irregular edges. Removing the irregularities will break the sequence of inner vertices into at most $2 c+1$ pieces. The graph induced by the largest of these pieces and the special vertices is $B_{n^{\prime \prime}}$. Assuming that the edges between inner vertices and $D$ are blue in the 2-coloring of $B_{n^{\prime \prime}}$ the transversal edges of $G_{\text {blue }}^{\prime \prime}$ and $G_{\text {red }}^{\prime \prime}$ are shown in Figure 8.
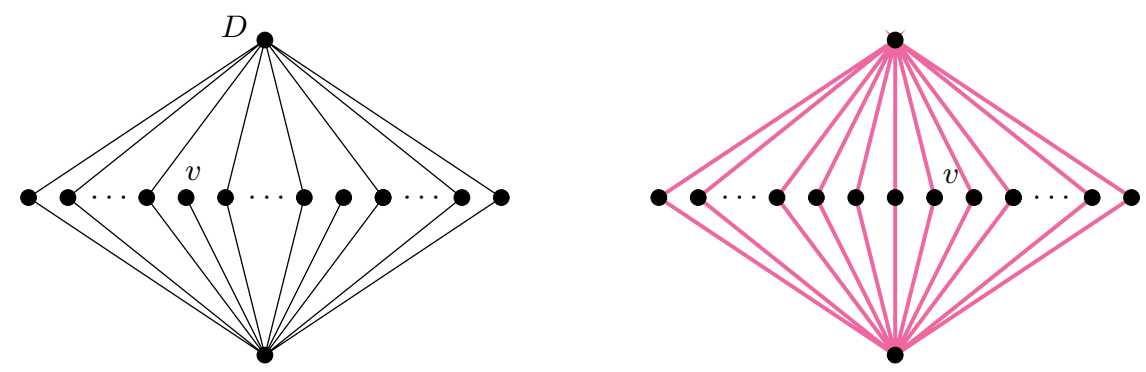

Fig. 8. Embedding of a reduced vertex $v$ in $G_{\text {blue }}^{\prime \prime}$ and $G_{\text {red }}^{\prime \prime}$

Let $v=v_{i-1}$ be a reduced vertex, the neighbors $v_{i}$ and $v_{i-3}$ of $v$ both have inner degree 5 . In $G_{\text {red }}^{\prime \prime}$ they have degree (at most) 2, hence, they must have degree 3 in $G_{\text {blue }}^{\prime \prime}$. This is only possible if $v_{i}, v_{i-1}$ and $v_{i-3}$ form a blue triangle. Since $v_{i-1}$ can have no further blue inner neighbors it follows that the edges $v_{i-1} v_{i-2}$ and $v_{i-1} v_{i+1}$ must be red.

Consider the edge $v_{i-2} v_{i-3}$. Suppose this edge is colored blue. To avoid closing a blue cycle, the edge $v_{i-2} v_{i}$ must be red. Then to avoid a red cycle the edge $v_{i} v_{i+1}$ must be blue. Continuing that way the colors of all edges to the right of the blue triangle in Fig. 9 are uniquely determined. To the other side consider the parity of blue and red edges at $v_{i-2}$, this forces $v_{i-2} v_{i-4}$ to be blue, while the parity at $v_{i-3}$ forces two red edges. To avoid a red cycle $v_{i-4} v_{i-5}$ must be blue, whence, parity forces $v_{i-4} v_{i-6}$ to be red. That way the color of edges left of the blue triangle is determined. The complete picture is shown in Fig. 9. We have found a blue Z-structure. 


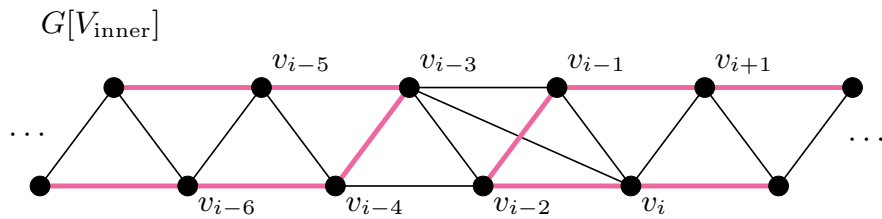

Fig. 9. The blue edge $v_{i-3} v_{i-2}$ implies a blue $\mathrm{Z}$

The other case where $v_{i-3} v_{i-2}$ is red leads, by similar arguments, to a red Z-structure.

We have seen that, given a modified vertex in $B_{n^{\prime \prime}}$, the parity condition and the cycle-freeness of the colored graphs induced by the inner vertices of $B_{n^{\prime \prime}}$ enforce a Z-structure. The zig-zag emanating from such a Z-structure in one direction has to run into the Z-structure of a second modified vertex. The outer paths of the other color make a turn at a modified vertex - and close a cycle at a second one. This is a contradiction since the blue triangles of the modified vertices are the only monochromatic cycles in $G\left[V_{\text {inner }}^{\prime \prime}\right]$. The contradiction shows that (for $n>25000$ ) there is no 2-coloring of $B_{n}$, hence, the thickness of the graph is 3 .

\section{Thickness of Semi Bar 1-Visibility Graphs}

Let $G=(V, E)$ be an SB1V given by a bar representation, see e.g. Fig. 2. In this section we present an algorithm which 2-colors the edges of $G$ such that each color class forms a plane graph in an embedding induced by the bar representation. Consequently the thickness of an SB1V is at most 2.

Between the full class of B1V graphs and the subclass SB1V there is the class of bar 1-visibility graphs admitting a representation by a set of bars such that there is a vertical line stabbing all bars of the representation. Note that the proof of the previous section implies that already in this intermediate class there are graphs of thickness 3 .

\subsection{One-Bend Drawing}

A one-bend drawing of a graph is a drawing in the plane in which each edge is a polyline with at most one bend. Here we introduce a one-bend drawing of semi bar 1-visibility graphs. This drawing is not planar in general, but it will be helpful for the construction and the analysis of the 2-coloring.

Enlarge the bars of each vertex $v$ to a rectangle $B(v)$ with a uniform width. Recall that we assume that the heights of all bars are different and that $B\left(r_{1}\right)$, $B\left(r_{2}\right), \ldots, B\left(r_{n}\right)$ lists the bars by decreasing height. Assign the stripe between the horizontal line touching the top of bar $B\left(r_{i}\right)$ and the horizontal line touching the top of bar $B\left(r_{i-1}\right)$ to $B\left(r_{i}\right)$; the dotted lines in Fig. 10 separate the stripes. Embed each vertex $v$ at the midpoint of the upper boundary of $B(v)$. We think of the edges as being directed from the longer bar (its starting bar) to the shorter bar (its ending bar). 


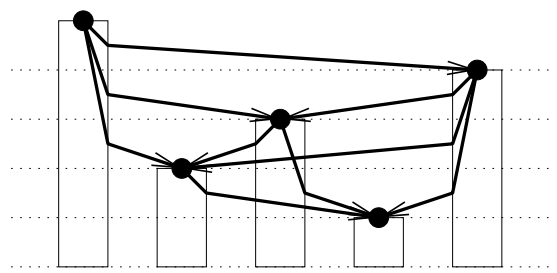

Fig. 10. The one-bend drawing of an SB1V

Now draw each edge $e=r_{i} r_{j}$ composed of two segments; the first segment is contained in $B\left(r_{i}\right)$, it connects $r_{i}$ with the inflection point $x_{e}$, the second segment connects $x_{e}$ with $r_{j}$ within the stripe of $r_{j}$. A good choice for $x_{e}$ which bewares from crossings between edges emanating from $r_{i}$ is to place $x_{e}$ on the vertical boundary of $B\left(r_{i}\right)$ which is closer to $r_{j}$ with a height which is inside the stripe of $r_{j}$. We call the segment $\left(r_{i}, x_{e}\right)$ the vertical part, the segment $\left(x_{e}, r_{j}\right)$ the horizontal part of the edge. Note that the stripe associated with $B(v)$ contains the horizontal parts of the incoming edges of $v$. Other edges might cross this stripe, but only with their vertical parts.

\subsection{2-Coloring Algorithm}

Now we present the algorithm 2PLANAR that provides a 2-coloring, i.e., a partition of the edges into two planar graphs (both on the vertex set $V$ ), using the given embedding. Thus, the algorithm produces planar embeddings of the two graphs such that each edge has only one bend. We think of the partition of the edges as a coloring with blue and red.

The idea of the algorithm is the following: Given the one-bend drawing, start with $r_{1}$, color all outgoing edges, move on to $r_{2}$, and so on. The algorithm uses an auxiliary coloring of the bars to determine the color of the edges.

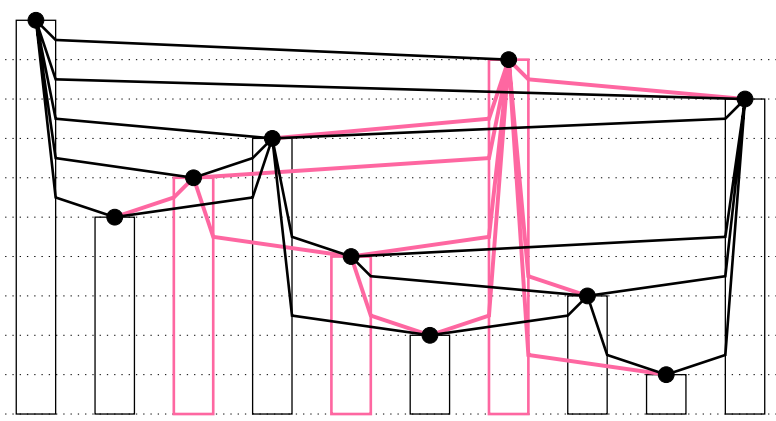

Fig. 11. A coloring produced by the algorithm 


\section{Algorithm 2PLANAR}

1. Start with $r_{1}$. Color $B\left(r_{1}\right)$ and all outgoing edges of $r_{1}$ blue. Whenever such an edge traverses another bar, color that bar red.

2. For $i=2, \ldots, n-1$

If $B\left(r_{i}\right)$ is uncolored, then color this bar blue.

For each uncolored edge $e=r_{i} r_{j}$

(a) If $e$ is a direct edge, it obtains the color of its starting bar $B\left(r_{i}\right)$.

(b) If $e$ is an indirect edge, check if the traversed bar has a color. If so, $e$ obtains the other color. Otherwise, it receives the color of its starting bar $B\left(r_{i}\right)$, and the traversed bar gets the opposite color.

Note that 2(b) implies the following:

Invariant. Whenever an edge traverses a bar the colors of the edge and the color of the bar are different.

Theorem 2. 2PLANAR produces a partition of $E$ into two plane edge sets.

Proof. We have to show that in the 2-coloring computed by 2PLANAR, any two crossing edges have different colors.

The one-bend drawing implies that crossings between edges only appear between the vertical part of one edge and the horizontal part of another edge. Consider a crossing pair $e, f$ of edges, assume that the crossing is on the vertical part of $e$ and the horizontal part of $f$. Hence, the crossing point is inside of the starting-bar of $e$, and the edge $f$ is an indirect edge traversing this bar (see Fig. 12).
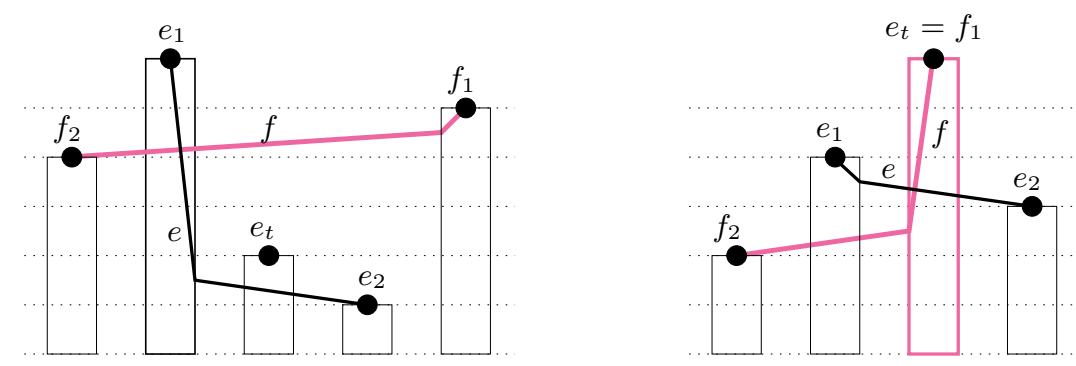

Fig. 12. Two crossing edges $e$ and $f$, shown in two possible configurations. Note that there can be many shorter bars between the bars depicted here.

Let the start-vertex of $e$ be $e_{1}$ and its end-vertex $e_{2}$. Similarly, let $f$ lead from $f_{1}$ to $f_{2}$. Suppose that the color of $f$ is red, then the invariant implies that $B\left(e_{1}\right)$ is blue.

If $e$ is a direct edge it obtains the color of its starting bar, in this case blue. Thus, assume that $e$ is an indirect edge. Then its color depends on the color 
of the traversed bar, let $B\left(e_{t}\right)$ be this bar. (Note that $e_{t}=f_{1}$ or $e_{t}=f_{2}$ are possible.)

The key for concluding the proof lies in the following lemma:

Lemma 2. If $B(v)$ is an arbitrary bar, then at most one longer bar can be the starting bar of indirect edges traversing $B(v)$.

Proof. Assume that $x=x_{1} x_{2}$ is an edge traversing $B(v)$ with $B\left(x_{1}\right)$ longer than $B(v)$, such that $B\left(x_{2}\right)$ is the shortest ending bar among all such edges. Then we know that between $B\left(x_{1}\right)$ and $B(v)$ in the left-to-right-order there can be no bar longer than $B\left(x_{2}\right)$, else it would block the line of sight corresponding to $x$.

Suppose there is another edge $y=y_{1} y_{2}$ starting from a bar $B\left(y_{1}\right)$ which is longer than $B(v)$. The choice of $x$ implies that the horizontal part of $y$ is above the horizontal part of $x$. Since $y$ can traverse only one bar it must connect to a bar $B\left(y_{i}\right)$ which is between $B(v)$ and $B\left(x_{1}\right)$ in the left-to-right-order. Since $y$ is above $x$ the bar $B\left(y_{i}\right)$ is longer than $B\left(x_{2}\right)$. This is in contradiction to the conclusion of the previous paragraph.

Now let's first assume that $B\left(e_{t}\right)$ is shorter than $B\left(e_{1}\right)$. Then by the lemma we know that $B\left(e_{1}\right)$ is the only longer bar sending an edge (e.g. the edge $e$ ) through $B\left(e_{t}\right)$. Therefore $B\left(e_{t}\right)$ is still uncolored when the algorithm considers $B\left(e_{1}\right)$, therefore, $e$ is colored with the color of $B\left(e_{1}\right)$, which is blue. This shows that the edges $e$ and $f$ have different colors in this case.

If $B\left(e_{t}\right)$ is longer than $B\left(e_{1}\right)$, then we can deduce $e_{t}=f_{1}$. For if a bar longer than $B\left(e_{t}\right)$ would be located strictly between $B\left(f_{1}\right)$ and $B\left(f_{2}\right)$ in the left-toright-order, the line of sight corresponding to $f$ would have to traverse two bars $\left(B\left(e_{1}\right)\right.$ and this longer bar), which is a contradiction. In addition, we know that $B\left(f_{2}\right)$ is shorter than $B\left(e_{1}\right)$, else $e$ and $f$ would not cross. Thus, we have $e_{t}=f_{1}$, and $B\left(f_{1}\right)$ is longer than $B\left(e_{1}\right)$. In this case the lemma tells us that $B\left(f_{1}\right)$ is the only longer bar sending an edge through $B\left(e_{1}\right)$. It follows that $B\left(e_{1}\right)$ was still uncolored when algorithm considered $B\left(f_{1}\right)$. Therefore, the red color of $f$ was chosen equal to the color of the bar $B\left(f_{1}\right)$. The invariant implies that the edge $e$, traversing the red bar $B\left(f_{1}\right)$, is blue. Hence, again $e$ and $f$ have different colors.

The algorithm shows that SB1Vs have graph-theoretical thickness not more than 2, and it provides a partition of the edges into two planar graphs, providing two plane embeddings. Since the edges are not straight lines, this does not show that the geometric thickness is bounded by 2. We think that any SB1V has a straight-line embedding such that the edges can be partitioned into two plane graphs. For emphasis we state this as:

Conjecture 1. Semi bar 1-visibility graphs have geometric thickness at most 2.

\section{Conclusion and Open Problems}

In this paper, we disproved the conjecture of Dean et al. 3. that the tight upper bound on the thickness of bar 1-visibility graphs is 2. We found a B1V with 
thickness 3, Dean et al. used the Four Color Theorem to show an upper bound of 4 - which still leaves a gap waiting to be closed. Considering that our quite sophisticated structure only yields some independent edges in the third planar layer, we make the following

Conjecture 2. The thickness of bar 1-visibility graphs is at most 3 .

On the way of getting a better understanding of bar $k$-visibility graphs we considered semi bar $k$-visibility graphs which have a strong combinatorial structure. Here, we proved a tight upper bound on the thickness for the case $k=1$. We can also use their structure to get tight bounds on the maximum number of edges, the chromatic number and the connectivity of SBkVs. Note for example that the shortest bar in a bar representation of an $\mathrm{SB} k \mathrm{~V}$ always corresponds to a vertex with degree at most $2(k+1)$, which provides a point of attack for inductions on the number of vertices.

These remarks are intended to give an idea of how semi bar $k$-visibility graphs promise to provide more approaches to attack problems about general bar $k$ visibility graphs. The following open questions may serve as a starting point for further research.

1. In [3], it is shown that the thickness of $\mathrm{B} k \mathrm{Vs}$ can be bounded by a function in $k$ (proven is a quadratic one). What is the smallest such function?

2. What is the largest thickness or geometric thickness of $\mathrm{SB} k \mathrm{Vs}$ ?

3. What is the largest chromatic number of $\mathrm{B} k \mathrm{Vs}$ ? Dean et al. show an upper bound of $6 k+6$.

4. Hartke, Vandenbussche and Wenger [8] found some forbidden induced subgraphs of $\mathrm{B} k \mathrm{Vs}$. They ask for further characterization of $\mathrm{B} k \mathrm{Vs}$ by forbidden subgraphs.

5. Hartke et al. also examined regular $\mathrm{B} k \mathrm{Vs}$. Are there $d$-regular $\mathrm{B} k \mathrm{Vs}$ for $d \geq 2 k+3 ?$

6. What is the largest crossing number of $\mathrm{B} k \mathrm{Vs}$ ?

7. What is the largest genus of $\mathrm{B} k \mathrm{Vs}$ ?

\section{References}

1. P. Bose, A. M. Dean, J. P. Hutchinson, and T. C. Shermer, On rectangle visibility graphs, in Proceedings of Graph Drawing '96, vol. 1353 of Lecture Notes Comput. Sci., Springer, 1997, pp. 25-44.

2. F. J. Cobos, J. C. Dana, F. Hurtado, A. Márquez, and F. Mateos, On a visibility representation of graphs, in Proceedings of Graph Drawing '95, vol. 1027 of Lecture Notes Comput. Sci., Springer, 1995, pp. 152-161.

3. A. M. Dean, W. Evans, E. Gethner, J. D. Laison, M. A. Safari, and W. T. Trotter, Bar k-visibility graphs. Manuscript, 2005.

4. - Bar k-visibility graphs: Bounds on the number of edges, chromatic number, and thickness, in Proceedings of Graph Drawing '05, vol. 3843 of Lecture Notes Comput. Sci., Springer, 2006, pp. 73-82. 
5. A. M. Dean, E. Gethner, And J. P. Hutchinson, Unit bar-visibility layouts of triangulated polygons., in Proceedings of Graph Drawing '04, vol. 3383 of Lecture Notes Comput. Sci., Springer, 2005, pp. 111-121.

6. M. B. Dillencourt, D. Eppstein, And D. S. Hirschberg, Geometric thickness of complete graphs, J. Graph Algorithms \& Applications, 4 (2000), pp. 5-17. Special issue for Graph Drawing '98.

7. D. Eppstein, Separating thickness from geometric thickness, in Proceedings of Graph Drawing '02, vol. 2528 of Lecture Notes Comput. Sci., Springer, 2002, pp. $150-161$.

8. S. G. Hartke, J. Vandenbussche, and P. Wenger, Further results on bar $k$ visibility graphs. Manuscript, November 2005.

9. J. Hutchinson, Arc- and circle-visibility graphs, Australas. Journal of Combin., 25 (2002), pp. 241-262.

10. J. P. Hutchinson, T. Shermer, And A. Vince, On representations of some thickness-two graphs, Comput. Geom. Theory Appl., 13 (1999), pp. 161-171.

11. A. Mansfield, Determining the thickness of graphs is NP-hard, Math. Proc. Camb. Phil. Soc., 9 (1983), pp. 9-23.

12. P. Mutzel, T. Odenthal, and M. Scharbrodt, The thickness of graphs: A survey, Graphs and Combinatorics, 14 (1998), pp. 59-73.

13. R. TAmassia And I. G. Tollis, A unified approach to visibility representations of planar graphs, Discrete Computational Geometry, 1 (1986), pp. 321-341.

14. S. K. Wismath, Characterizing bar line-of-sight graphs, in Proceedings of SCG '85, ACM Press, 1985, pp. 147-152. 